\title{
Optimization of Armored Equipment Maintenance Material Regulating based on Gray Entropy
}

Hao LI

Academy of Armored Force Engineering

Beijing, China

E-mail:sddy1990@yeah.net

Long-Tao WU

Academy of Armored Force Engineering

Beijing, China

E-mail:673729039@qq.com

Yong-Fei MEI

Academy of Armored Force Engineering

Beijing, China

E-mail:meiyongfei1985@126.com

\author{
Tie-Ning WANG \\ Academy of Armored Force Engineering \\ Beijing, China \\ E-mail:wtn0728@163.com \\ Zhao-Jie CHEN \\ Academy of Armored Force Engineering \\ Beijing, China \\ E-mail:843364390@qq.com
}

\begin{abstract}
Most of the armored equipment maintenance material regulating is passive application from the junior resource, and the superior resource is lack of the initiative conduction. Through the import of Gray entropy, establish the dynamic requirement model, not only measure the material demands effectively, but also bring forward the response origin point. At last validate its rationality with the example, providing a new thinking for optimizing the material scheduling process.
\end{abstract}

Keywords-armored equipment; maintenance material; regulate; gray entropy

\section{INTRODUCTION}

Requirements of the armored equipment maintenance materials from consumptive unit are indeterminacy, once the material is under-reserve, regulation is needed: literature[1]regards the cost-control as the target, establish the supply support model based on internal-region and cross-region regulate; literature[2] establishes the generalized optimum path and transport models of emergency material regulate-decision, in order to improve the response-speed of materiel regulation, literature[3] designs the material regulation system, all of the above-mentioned literatures improve the scientificity of material schedule-decision, but the optimization of the response origin point is neglected.

In order to simplify the dispose process, the paper regards the troop-resource and the corresponding consumptive unit as the only study object, through the introduction of the gray entropy and establishment of the dynamic requirement model, requirement of the material is estimated accurately, and bring forward the regulation origin point, and the regulation time-consuming is shortened for essence, improving the initiative action ability of the superior resource, and then the regulation process is optimized.

\section{ANALYSIS OF THE ARMORED EQUIPMENT MATERIAL REGULATION}

Material support is composed by series of incidents (consume, produce, apply, deliver and deliver) and some processes with the characteristic of time span (produce process, consume process and transport process), the regulation process is shown as follows [4]: 


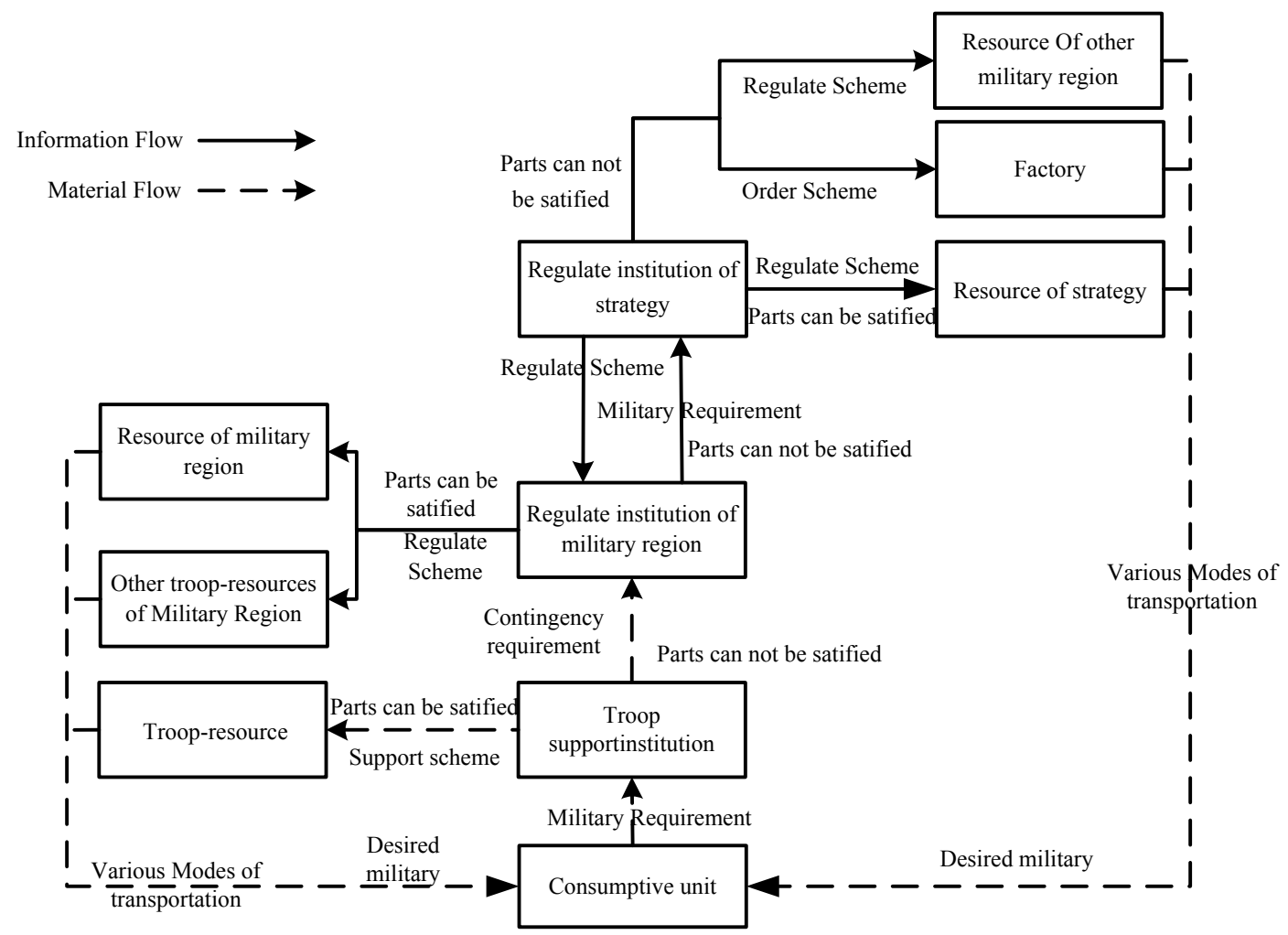

Figure 1. Flowchart of the Material Regulate

It can be known that the material regulation process can be divided into two parts:

(1) Material requirement is generated from the consumptive unit, uploaded with the message, and accompanied the disposed of the superior institution.

(2) The message transmits to lower levels, with the material regulation.

In the realistic regulation, it consumes much time to upload and transmit of the message, influencing the train plan of the consumptive unit at a certain degree, forecasting the schedule requirement timely and effectively, and it is the key to improve the material support level to form the protract time that can buffers the influence of the time-postponing.

\section{ESTABLISHMENT OF DYNAMIC REQUIREMENT MODEL BASED ON GRAY ENTROPY}

Process of material regulation is typical discrete event based on limited message space, as the functions to measure the extent between the isolated material system and balance system, gray entropy can dispose the discrete event effectively, and with the smaller of the value of number, the extent becomes larger to departure balance system.

\section{A. Gray Entropy}

Assume that the discrete sequence which exists in limited message space $X=\left\{x_{i} \mid i=1,2, \cdots \quad \forall i, x_{i} \geq 0\right.$ and $\sum_{i=1}^{n} x_{i}=1$, then the $\mathrm{H}(\mathrm{X})$ can be called the Gray Entropy of

$\mathrm{X}$ [5], and the stipulation is shown as follows:

$$
\left\{\begin{array}{l}
H(X)=-\sum_{i=1}^{n} x_{i} \ln x_{i} \\
0 \ln 0 \hat{0}
\end{array}\right.
$$

The material regulation requirement of the troop resource can be assessed rationally by the introduction of the gray entropy, in order to simply the dispose process, stipulate as follows:

(1) Assume that the fund is sufficient, and the influence of transport capacity and constraint is not considered;

(2)Neglect the assembly units' interchangeability between different equipment, because of the difference of the equipment's in consumptive unit and the bad university.

The establishment of dynamic requirement model based on gray entropy, is the situation to overall the equipment's' material schedule requirement in consumptive unit, and it can not only measure the support level, but also explicit its schedule limit.

\section{B. Establishment of Maintenance Material Requirement Model}

Through the statistical lifetime analysis of armored equipment parts, most of their forms can be reduced to three 
types: Poisson distribution, Gaussian distribution and Weibull Distribution. In order to simplify the treating processes, the further study is made only for the parts that belongs to these three types.

(1) Poisson Distribution

When one part's failure rate is the steady state value, its requirement for the maintenance material is also the steady state value, and can be described with the Poisson distribution:

$$
P\left(W_{1}\right)=\sum_{x=0}^{W_{1}} \frac{(\alpha L t)^{x}}{x !} e^{-\alpha L t}
$$

In the formula:

$t$-the part's serive time, $x$-the maintenance material quantity which can be offered when the part is breakdown, $P\left(W_{1}\right)$-the guarantee level of the maintenance material, $\alpha$ -the probability of the part to be breakdown, $L$-the fitting quantity of one part, $W_{1}$-the intending requirement of maintenance material to satisfy the guarantee level.

(2)Gaussian distribution

When one part's working life is constant duration relatively, the requirement W2can be described with Gaussian distribution:

$$
W_{2}=\frac{t}{E}+\mu_{\rho} \sqrt{\frac{\sigma^{2} t}{E^{3}}}
$$

In the formula:

$E$-the part's lifetime mean value, $\sigma$-standard deviation of the part's lifetime, $\mu_{\rho}$-tantile.

(3)Weibull Distribution

When one part's lifetime owns the characteristic of 'tub curve', the requirement W2can be described with Weibull distribution:

$$
W_{3}=\left(\frac{\mu_{\rho} k}{2}+\sqrt{\left(\frac{\mu_{\rho} k}{2}\right)^{2}+\frac{t}{E}}\right)^{2}
$$

In the formula:

$E=\eta \Gamma\left(1+\frac{1}{\beta}\right), \quad \beta$ is shape parameter, $\eta$ is scale parameter, $\mathrm{k}-$ variation coefficient, and

$$
k=\sqrt{\frac{\Gamma\left(1+\frac{2}{\beta}\right)}{\Gamma\left(1+\frac{2}{\beta}\right)^{2}}-1}
$$

With the equipment's test data and the monitor data in using, describe the requirement of maintenance material quantification ally, which the optimization of the schedule point is built on.

\section{Establishment of the Resource's Dynamic Requirement}

In order to establish the model commodiously, confine the study to the constant type of the equipment belongs to consumptive unit, and the essential symbol hypothesis is shown as follows:

For the equipment's whose quantity is $P$, the time gather put to use $T=\left\{T_{1}, T 2, T 3, \ldots, T_{y}, \ldots T_{p}\right\}$;

Parts gather in the equipment whose lifetime forms accord with the poisson distribution $N_{1}=\left\{n_{1}, n_{2}, n_{3}, \ldots, n_{s}\right\}$, the material gather needed that are used to recover the technical conditions $W_{1}=\left\{w_{1}, w_{2}, w_{3}, \ldots, w_{s}\right\}$, the material in store gather $M_{1}=\left\{m_{1}, m_{2}, m_{3}, \ldots, m_{s}\right\}, \quad$ and $s$ is the statistical magnitude of the parts vatiety conform to this distribution;

Parts gather in the equipment whose lifetime forms accord with the Gaussian distribution $N_{2}=\left\{n_{s+1}, n_{s+2}, n_{s+3}, \ldots, n_{s+q}\right\}$, the material gather needed that are used to recover the technical conditions $W_{2}=\left\{w_{s+1}, w_{s+2}, w_{s+3}, \ldots, w_{s+q}\right\}$, the material in store gather $M_{2}=\left\{m_{s+1}, m_{s+2}, m_{s+3}, \ldots, m_{s+q}\right\}$, and $q$ is the statistical magnitude of the parts vatiety conform to this distribution;

Parts gather in the equipment whose lifetime forms accord with the weibull distribution $N_{3}=\left\{n_{s+q+1}, n_{s+q+2}, n_{s+q+3}, \ldots, n_{s+q+r}\right\}$, the material gather needed that are used to recover the technical $W_{3}=\left\{w_{s+q+1}, w_{s+q+2}, w_{s+q+3}, \ldots, w_{s+q+r}\right\}$, the material in store gather $M_{3}=\left\{m_{s+q+1}, m_{s+q+2}, \ldots, m_{s+q+r}\right\}$, and $r$ is the statistical magnitude of the parts variety conform to this distribution; $\mathrm{Tx}$ :

Then the requirement of material $\mathrm{x}$ in the time window

(1)The requirement of part $\mathrm{j}(1 \leq j \leq s)$ whose lifetime forms accord with the Poisson distribution:

$$
P\left(w_{y 1 j}\right)=\sum_{f=0}^{w_{1 j}} \int_{0}^{T} \frac{\left(\alpha_{1 j} L_{1 j} t_{y 1 j}\right)^{f}}{n_{y 1 j} !} e^{-\alpha_{1 j} L_{1 j} t_{y 1 j}} d t
$$

(2)The requirement of part $j(s+1 \leq j \leq s+q)$ whose lifetime forms accord with the Gaussian distribution:

$$
w_{y 2 j}=\int_{0}^{T_{x}}\left(\frac{t_{y 2 j}}{E_{2 y 2 j}}+\mu_{2 j \rho} \sqrt{\left.\frac{\sigma_{2 j}{ }^{2} t_{y 2 j}}{E_{2 y 2 j}{ }^{3}}\right)} d t\right.
$$

(3)The requirement of part $j(s+q+1 \leq j \leq s+q+r)$ whose lifetime forms accord with the weibull distribution:

$$
w_{y 3 j}=\int_{0}^{T_{x}}\left(\frac{\mu_{3 j \rho} k_{y 3 j}}{2}+\sqrt{\left(\frac{\mu_{3 j \rho} k_{y 3 j}}{2}\right)^{2}+\frac{t_{y 3 j}}{E_{y 3 j}}}\right)^{2} d t
$$

So, the equipment's material requirement can be calculated: Requirement of parts whose lifetime forms accord with the Poisson distribution is 
$w_{1 j}=\sum_{y=1}^{P} w_{y 1 j} ;$ Requirement of parts whose lifetime forms accord with the Gaussian distribution is $w_{2 j}=\sum_{y=1}^{P} w_{y 2 j}$; Requirement of parts whose lifetime forms accord with the weibull distribution is $w_{3 j}=\sum_{y=1}^{P} w_{y 3 j}$.

Then the material deficiency can be known:

$$
\Delta w_{j}=\left\{\begin{array}{l}
m_{1 j}-w_{1 j}, \text { when } 1 \leq j \leq s \\
m_{2 j}-w_{2 j}, \text { when } s+1 \leq j \leq s+q \\
w_{3 j}-m_{3 j}, \text { when } s+q+1 \leq j \leq s+q+r
\end{array}\right.
$$

If $\Delta w_{j} \geq 0$, it indicates that the stored material can meet the requirement of the consumptive unit; if $\Delta w_{j}<0$, it indicates that the stored material cannot meet the requirement of the consumptive unit, then the regulation is needed:

Gray Entropy is used to measure the extent to diverge the balance system, if the stored material can meet the requirement of the consumptive unit, then the balance is not influenced, so $\Delta w \ln \Delta w=0$, so the object should be confined to the material which $\Delta w<0$, but the element in gray entropy should be guaranteed $\forall i, x_{i} \geq 0$, so the further managing step is needed: (1) Ensure the total material quantity for applying

$$
W=\sum \Delta w_{j}
$$

(2) Normalize and calculate the distance with the climax, and normalize again

$<1>$ First time to normalize

$$
\Delta w_{j}{ }^{\prime}=\Delta w_{j} / W
$$

$<2>$ calculate the distance

$$
d_{j}=\left|\Delta w_{j}{ }^{\prime}-\frac{1}{e}\right|
$$

$<3>$ Normalize again

$$
\Delta w_{j}{ }^{\prime}=d_{j} / \sum d_{j}
$$

(3) Calculate the gray entropy in consumptive unit

$$
H(G)=-\sum \Delta w_{j} " \ln \Delta w_{j} "
$$

Parts of the maintenance materials stored is over-stocked, so the constitution of the gray entropy corresponding parts influence the equipment operation seriously, but limited by the length, only introduce the significance factor to distinguish the gray entropy, so that the materials' schedule dynamic requirement of troop-resource $R(G)$ can be calculated:

$$
R(G)=H(G)+\varphi W
$$

\section{ANALYSIS OF EXAMPLES}

Protract possibility of the schedule origin has been explained in 2.2, and because of the difficulty to acquire the trial run data, the quantification duration is hard to calculate, so just make a simple example: Assume that there are three troop-resources $G_{1}, G_{2}$ and $G_{3}$ supported by the same superior resource, and the corresponding consumptive unitsare $A_{1}, A_{2}, A_{3}$,through monitoring and analyzing of the data from the parts $n_{1}$ and $n_{2}$ between Jan,2014 and June,2014, the materials' requirement $x_{11}, x_{12}, x_{21}, x_{22}, x_{31}, x_{32}$ can be calculated, quantity and the inventory is shown as follows:

TABLE I. MATERIALS' REQUIREMENT AND THE INVENTORY DATA

\begin{tabular}{ccccc}
\hline $\begin{array}{c}\text { consumptiv } \\
\text { e unit }\end{array}$ & $\begin{array}{c}\text { materia } \\
\text { 1 }\end{array}$ & $\begin{array}{c}\text { requirement(piec } \\
\text { e) }\end{array}$ & $\begin{array}{c}\text { resourc } \\
\mathrm{e}\end{array}$ & $\begin{array}{c}\text { inventory(piece } \\
\text { A }\end{array}$ \\
\hline \multirow{2}{*}{$A_{1}$} & $x_{11}$ & 380 & \multirow{2}{*}{$G_{1}$} & 352 \\
& $x_{12}$ & 456 & & 426 \\
\hline \multirow{2}{*}{$A_{2}$} & $x_{21}$ & 313 & $G_{2}$ & 285 \\
& $x_{22}$ & 460 & & 443 \\
\hline \multirow{2}{*}{$A_{3}$} & $x_{31}$ & 359 & $G_{3}$ & 339 \\
& $x_{32}$ & 452 & & 422 \\
\hline
\end{tabular}

The decision process of material regulation is:

(1) Ensure the total material quantity of junior resource for regulating

$$
\begin{gathered}
W_{1}=\sum_{j=1}^{2} \Delta w_{j}=(380-352)+(456-426)=58 \\
W_{2}=\sum_{j=1}^{2} \Delta w_{j}=(313-285)+(460-443)=45 \\
W_{3}=\sum_{j=1}^{2} \Delta w_{j}=(359-339)+(452-422)=50
\end{gathered}
$$

(2)Normalize

$$
\Delta w_{11} "=0.435, \Delta w_{12} "=0.565
$$




$$
\begin{aligned}
& \Delta w_{21} "=0.963, \Delta w_{22} "=0.037 \\
& \Delta w_{31} "=0.122, \Delta w_{32} "=0.878
\end{aligned}
$$

(3)Calculate the gray entropy

$$
H\left(G_{1}\right)=0.685, H\left(G_{2}\right)=0.158, H\left(G_{3}\right)=0.449
$$

Then the requirement of troop-resource can be quantified as follows:

$$
\begin{aligned}
& R\left(G_{1}\right)=H\left(G_{1}\right)+\varphi W_{1}=0.685+58 \varphi \\
& R\left(G_{2}\right)=H\left(G_{2}\right)+\varphi W_{2}=0.158+45 \varphi \\
& R\left(G_{3}\right)=H\left(G_{3}\right)+\varphi W_{3}=0.449+50 \varphi
\end{aligned}
$$

So, $\mathrm{G} 1>\mathrm{G} 3>\mathrm{G} 2$, it indicates that the requirement of $\mathrm{G} 1$ is the most pressing, and should be satisfied at first.

\section{CONCLUSIONS}

This paper establishes the requirement model based on the relevance between the parts with different lifetime forms and its requirement, through the introduce of the gray entropy, the paper measures the requirement quantitative, affording the scientific gist for the decision of dispatch, and the protract of the dispatch origins realized, with the process optimizing, the possibility of superior resource's initiative action is improved at a certain extent.

\section{REFERENCES}

[1] KE Rong-bo,WANG Tie-ning,YUShuang-shuang. Decision Model of Armored Equipment Regulating Support[J].Journal of Academy of Armored Force Engineering, 2014,28(1):21-23.

[2] WANG Tie-ning,LIANGBo,CAOYu,etc. Decision Model of Equipment Materiel Emergency Dispatch Based on Multi-resource Points[J].Journal of Academy of Armored Force Engineering, ,2012,26(6):10-14.

[3] WANG Sheng-de, LI Qing-quan.Study of Equipment material dispatchSystem[J]China Logistics \& Purchasing,2015,02:76-77.

[4] ZHANG Wen-jun.Research on Material Support Resource Scheduling Optimization[D].Beijing: Academy of Armored Force Engineering,2007.

[5] WANG Zheng-xin,DANG Yao-guo,CAO Ming-xia. Weighted degree of grey incidence based on optimized entropy[J].Systems Engineering and Electronics,2010,04:774-776. 